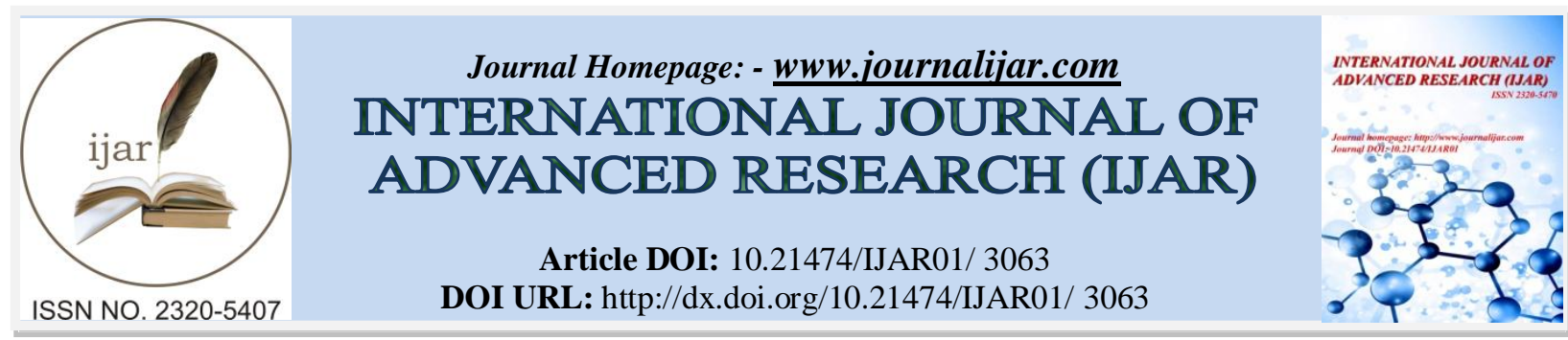

RESEARCH ARTICLE

\title{
MAIN PREDICTORS AND TREATMENTS OF PERI-IMPLANTITE: BRIEF REVIEW
}

Edmiane Fernanda Batista Monteiro de Carvalho ${ }^{1}$, Tacyane Petrolli Alberici ${ }^{1}$, Mariluciade Moraes Alves Moreira $^{1}$, Ana Paula Bernardes da Rosa Maluf Abbud ${ }^{1,2}$, Carlos Alberto Costa Neves Buchala ${ }^{1,2}$,Elias Naim Kassis and Idiberto Jose Zotarelli Filho ${ }^{1,2}$

1. University Center North Paulista (Unorp) - Sao Jose do Rio Preto - SP, Brazil.

2. Post graduateandcontinuingeducation (Unipos), Street Ipiranga, 3460, São José do Rio Preto SP, Brazil 15020040.

\section{Manuscript Info}

\section{Manuscript History}

Received: 03 December 2016

Final Accepted: 30 December 2016

Published: January 2017

Key words:-

Peri-implantite, Osteointegration, Mucositis and Implants.

\section{Abstract}

Background: About 3 million Americans have dental implants, and this number is growing at 500,000 every year. In this context, the microorganisms that reside on the surfaces of the implants and their prosthetic components are considered the main etiological factor for the peri-implantite.

Objective: was todo a brief literary review, pointing out the main predictors of pri-implantite and treatments. Methods: This study is in the literature review focusing on the main predictors and treatments of peri-implantite. To this end, a survey was conducted in scientific articles in PubMed, Medline, Scielo, Lilacs and books.

Discussion: Several uncertainties remain regarding peri-implant disease. Long-term follow-up of consecutively treated cases appears to be a realistic path for accumulating more information. The current clinical recommendations are that there is no consensus on the protocol for the treatment of mucositis or peri-implantitis due to the heterogeneous characteristics of different implant systems, implant position and other factors related to the patient. Thus, there is no individual treatment option. Conclusion: In the implant, if plaque accumulation is allowed for longer periods and the patient is at risk, peri-implant mucositis can progress to lesions with peri-implantite alveolar bone loss. The most important treatment of peri-implantitis is to interrupt progressive bone loss by plaque control, even restoring the bone lost through regenerative surgical procedures.

Copy Right, IJAR, 2016,. All rights reserved.

\section{Introduction:-}

About 3 million Americans have dental implants, and this number is growing at 500,000 every year. In addition, the number of biological complications is also increasing. Among them, peri-implant disease is considered the most common cause of implant loss after osseointegration. In this context, the microorganisms that reside on the surfaces of the implants and their prosthetic components are considered the main etiological factor for peri-implantite [1,2].

Periodontal and peri-implant soft tissues have many characteristics in both clinical and histological [1-3]. For proper control and maintenance of these tissues, a better understanding of the differences and similarities that exist between 
them is needed. Thus, biofilms are formed on all adherent surfaces in a fluid system, both in teeth and in oral implants. As a result of bacterial presence, the host responds by developing a defense mechanism that will lead to soft tissue inflammation [3,4]. In the dento-gingival unit, the results are gingivitis lesions [3].

Furthermore, experimental research has shown that mucositis can progress to peri-implantitis, depending on the factors and risk indicators, affecting the peri-implantar support bone circumferentially [5,6]. Although the supporting bone is lost coronally, the implant will still remain integrated bone and therefore clinically stable. This is the reason why mobility represents a diagnostic characteristic of peri-implantitis that is not sensitive but more specific [6].

More sensitive and reliable parameters of the development and presence of peri-implant infections are bleeding probing, depth of probing and radiographic interpretations of radiographs, aiming to detect the peri-implant lesions as early as possible, thus enabling the treatment, which in this review is described As a cumulative procedure, starting with simpler procedures until the most advanced, aiming to paralyze and prevent the progression of these lesions, which would inevitably result in failure of the implant $[5,6]$.

In this context, most publications from 2011 to 2015 evaluated the effect of implant surface roughness on long-term bone loss as a substitute for peri-implantitis risk. 87 of the 2,566 studies reported average bone loss after at least 5 years. The prevalence of implants with bone loss ranging from 1.0 to $3.0 \mathrm{~mm}$ was estimated. Bone loss was used as a surrogate parameter for peri-implantitis, since the prevalence of peri-implantitis was not reported in most studies or when reported, the diagnostic criteria were unclear or of doubtful quality [1- 6].

Perimplant bone loss around minimally harsh implant systems was statistically less significant compared to moderately rough and rough implant systems [2]. There was no statistically significant difference between moderately rough and rough implant systems [2]. Studies comparing implants with comparable design and different surface roughness, showed lower perimplantar mean bone loss around the less rough surfaces in the meta-analysis. In addition, when considering $3.0 \mathrm{~mm}$ of bone loss after at least 5 years to represent the presence of "periimplantite", less than $5.0 \%$ of the implants were affected. Still, periodontal history and smoking habits were the most important predictors of bone loss [4].

Therefore, the present work aimed to make a brief literary review, pointing out the main predictors of pri-implantite and treatments.

\section{Methods:-}

This study is in a literature review focusing osprincipaispreditores e tratamentos de peri-implantite. To this end, a survey was conducted in scientific articles in the databases PubMed, Medline, Scielo, Lilacs and books. The key words used were Peri-implantite, Osteointegration, Mucosite e Implants, which are registered in the Health Sciences Descriptors. Selected papers passed criteria for inclusion and exclusion.

\section{Main Predictors Continuous or Categorical:-}

The main predictors are periodontal history, smoking habits, osteoporosis, diabetes mellitus.

\section{MainPredictorsAnswer:-}

The main predictor of response was peri-implantite.

\section{Literature Review:-}

The peri-implantite is developed from mucositis lesions, while others remain resistant and present only mucositis in response to the accumulation of plaque microorganisms [1-3]. The term periimplantite is used because of clinical, histological and microbiological similarities with periodontitis. Several studies suggest that destruction of periimplant tissues is caused by a bacterial infection and that the concomitant inflammation observed is similar to periodontal disease [7,8]. Peri-implantitis is defined as an inflammatory process that affects the tissues around an osseointegrated implant as a result of the progressive loss of peri-implant bone support accompanied by inflammatory soft tissue disease [6-9].

As the tissues around teeth and implants have many characteristics in common it is inevitable that the host bacterium interaction also plays a potential role in peri-implant complications, as well as in periodontitis [6]. Gualini and 
Berglundht (2003) [9] investigated the histochemical characteristics of the peri-implant tissues during mucositis and peiiimplantar disease. In order to do so, they selected two groups of studies: in group A (mucositis) ten patients partially treated with branemark implants as a function of $+/$ - five years. In group B (periimplantite) six patients partially restored also with branemark type implants in use at $+/$ - eleven years. In each group at sites with periimplant disease, biopsies ( $4 / \mathrm{mm}$ of soft tissue) were performed and analyzed. Before the biopsies, patients were not on any periodontal treatment program.

In addition, restorations supported by dental implants have been added substantially to the clinical treatment options presented to patients [3]. However, complications with these treatment options also arise due to inadequate patient selection and inadequate planning of treatment combined with poor follow-up care [4]. Complications related to the presence of inflammation include perimucositis, peri-implant bone loss, and peri-implantitis. The prevalence rates of these complications were reported to be as high as 56.0\% [4]. Treatment options that have been reported include non-surgical therapy, the use of antibiotics administered locally and systematically, and surgical protocols aimed at regenerating lost bone and soft tissue around implants [5].

In another study Esposito et al. (1997) [10], studied the peri-implant soft tissues of implants lost after being in use for a certain period. They described the cellular composition of soft tissues that were around implants, which lost osseointegration (they were soft) and were removed the stable and healthy implants were used as control. In the research the lost implants were divided into two groups: those that were lost with infection and those without infection. The group with infection (perimplantite) were characterized by an intense inflammatory and immunological response [10].

The connective tissue had an inflammatory infiltrate containing a large number of plasma cells (predominant cells) and mono-nuclear cells (B and T lymphocytes, monocytes and macrophages). There was migration of the sulcular epithelium and in its interior the presence of neutrophils PMNs and in some cases the apical migration of the epithelium circumvented every implant $[4,11]$. Because of these characteristics, the authors suggest that peri-implant soft tissues react to bacterial invasion with similarity to periodontal tissues. The presence and distribution of soft tissue macrophages around lost implants were similar to those found in the control group of healthy implants [12$15]$.

Thus, the presence of macrophages around the tissues of the implant is a phenomenon related to the presence of a biomaterial. The macrophage is the most common cell type detected on the implant surface, regardless of the type of material, and has been suggested that it plays a key role during the inflammatory and repair phase after implantation [3,7]. According to Liljenberg et al. (1994) [11] the presence of a large number of macrophages seems to be different in periodontal disease active or inactive in humans as well as experimentally in animals.

Experimental evidence has shown us that failed implants do not appear to be distributed randomly in the population, but concentrated in a small percentage of the population. Weyant and Burt (1993) [13] studied the survival of dental implants in a group of five hundred and ninety-eight consecutive patients from the U.S. dental implant veterinary administration file, totaling two thousand ninety-eight implants. Their results indicate that, of all the group, a total of eighty-one implants of forty-five individuals were removed between five and a half years.

Smoking that alter immune functions and tissue repair or endogenous or intrinsic factors such as genetic predisposition for a hyper cytokine production, low antibody production or a decrease in the number of neutrophils can cause large changes in the disease process [14]. These factors that can modify the host response, alter the susceptibility of the individual to infection by the periodontal microorganisms are the risk factors.

\section{Main Predictors:-}

Smoking:-

Smoking alters the balance between the microbial load on the implants and the host response, negatively impacting the longevity of oral implants [2]. The mechanisms by which smoking affects peri-implant tissues are immunosuppressive effects in the host. Smokers also have low levels of neutrophils [14]. Pathogenic periodontii may now escape the specific and nonspecific mechanisms of immune cleansing, allowing them to establish themselves as peri-implant subgingival inhabitants. Thus, the alteration in the subgingival physical environment and the decreased oxygen tension will allow the growth of an anaerobic microbiota. In addition, smoking increases 
bacterial adhesion to epithelial cells. However, it is not surprising that smokers in general have higher levels of periodontospathogens and may be considered microbiologically compromised [15].

Thus, in an effort to evaluate possible risk factors that would contribute to implant failure, Bain and Moy (1993) [16] conducted a retrospective evaluation on two thousand ninety-four branemark implants, installed by a surgeon between one thousand Nine hundred and eighty-four and one thousand nine hundred and ninety-one. Values considered included length, location, diabetes, medication with steroids age and sex, time placed along the "learning curve" and use of cigarette. It soon became clear that of all factors considered, smoking took the most significant part in the high failure rate of implants. Their results showed an overall failure rate of $4.76 \%$ in nonsmokers versus $11.3 \%$ in smokers.

In addition, when only the maxilla was considered, there was a failure of $17.90 \%$ in smokers and only $7.3 \%$ in nonsmokers. The difference in the mandible was lower, with $4.64 \%$ of failure in smokers and $2.4 \%$ in non-smokers, with significant difference only in the anterior mandible [16]. The failure rates of implants decreased with their increased length, but in the maxilla there was a more significant failure rate in smokers of up to $15 \mathrm{~mm}$ [17]. Haas et al. (1996) [18] evaluated the maxillary gingival bleeding index, a periimplantar probing rate and peri-implantar inflammation, as well as mesial and distal bone loss, higher in smokers than in nonsmokers.

Moreover, Lindquist et al (1997) [17] found that the marginal loss was higher in smokers than in nonsmokers and correlated with the amount of cigarette consumption. Smokers with poor oral hygiene showed greater marginal loss around lower implants than those with good oral hygiene.

\section{Oral hygiene:-}

The oral hygiene of the patient has an important significance in the stability of the marginal bone around osseointegrated implants. A higher prevalence of peri-implant problems has been reported in patients with low oral hygiene index. Even in fully edentulous patients with dental implants, ineffective oral hygiene has been linked to increased peri-implant bone loss especially in smokers [18].

\section{Susceptibility to periodontitis:-}

Implants can be affected by periodontal conditions comes from two evidences: similarity in the microflora that colonizes teeth and implants in partially edentulous patients and identification of a hyperinflammatory phenotype in partially edentulous patients with peri-implant lesions [3]. Fardal et al. (1999) [19] described an unsuccessful therapy with implants in a partially edentulous patient suffering from aggressive periodontitis. However, authors try to prove the opposite and point out that some implant and surface configurations may be even more resistant to "insertion" loss than teeth. Thus installed implants, especially those with rough surfaces, in patients prone to aggressive periodontitis may lead to a high risk of implant loss [20].

\section{Osteoporosis:-}

Osteoporosis may be a risk factor for oral bone loss, including the alveolar process associated with periodontal infection [5]. Wowren et al. (1994) [21] showed a relationship between periodontal disease and osteoporosis, comparing twelve women with fractures due to osteoporosis with fourteen normal women, and found more loss of periodontal insertion in women with osteoporosis than in those without osteoporosis.

Although some studies Wactawski et al. (1996) [22] point to the possibility of osteoporosis without a risk factor for periodontal disease and peri-implantar disease and other studies are needed especially those on a large scale where multiple risk factors affect both osteoporosis and periodontal disease.

\section{Diabetes:-}

It is well established that diabetic patients are more prone to healing complications even in clean wounds $[2,3]$. There is a number of evidences supporting the association between diabetes mellitus and periodontal disease. Taylor et al. (1998) [23] in a longitudinal study showed that type II diabetes was a significant risk factor for periodontal disease. 
Table 1:- Main considerations regarding the treatment of peri-implantitis [6].

1. Non-surgical treatment appears to be more effective for the treatment of mucositis than peri-implantitis.

2. Peri-implantite treated with non-surgical therapy results mainly in decreased bleeding probing and depth of probing (usually less than $1 \mathrm{~mm}$ ).

3. Personal hygiene care or the professional maintenance program have a positive effect on the prevention of peri-implant mucositis that begins in peri-implantitis.

4. The implant of non-regenerative surgical therapy should be established based on the diagnosis and risk profile.

Table 2:- Prediction of improvement of clinical parameters that apply peri-implantite regenerative surgical treatment [6].

1. The evaluation of the systemic and local factors of the patients affected by the perimplantite should be taken into consideration in the application of the regenerative surgical treatment.

2. Regenerative surgical treatment may be chosen for the reconstruction of intra-auricular defects, while the nonregenerative approach and implantoplasty of the implant component are recommended.

3. Adequate maintenance phases of pre and post-surgical hygiene and effective decontamination of the implant surface are required for the successful surgical procedure.

4. There is no fundamental advantage of using the membrane to cover the bone graft in the final result of the regeneration of peri-implant defects.

5. Immersion of the implants during the healing period does not appear to influence the final outcome of the regenerative treatment.

\section{Discussion:-}

Several uncertainties remain regarding peri-implant disease. Long-term follow-up of consecutively treated cases appears to be a realistic path for accumulating more information [1]. This can be useful in establishing the magnitude and stability presitivity on the improvements that can be made. Although not generally reported, it appears that bandage-induced defects are scintillating and funnel-shaped [2].

The current clinical recommendations are that there is no consensus on the protocol for the treatment of mucositis or peri-implantite due to the heterogeneous characteristics of different implant systems, implant position and other factors related to the patient 3. Thus, there is no individual treatment option. Table 1 lists the main considerations regarding peri-implantite treatment [6].

There is still no consensus on a particular protocol or biomaterial selection in the peri-implantite regenerative surgical treatment due to the high heterogeneity and bias among the investigated studies. However, several clinical recommendations can be elaborated according to the data available in the current literature. A predictable improvement of the clinical parameters that apply to the peri-implantite regenerative surgical treatment can be predicted, as shown in table 2 [6]. In light of the peri-implant microbial etiology, the motivation and instruction of the patient together with the surgical therapy envelope are an integral part of the peri-implantite treatment. No specific clinical recommendation can be made as to which specific method of non-regenerative surgical therapy is to be implemented [6].

The formation of new bone in the apical part of the defects may have occurred against a surface that was not contaminated prior to treatment [24]. However, the magnitude of the new bone formation obtained in some studies suggests that new bone can be formed in direct contact with a previously contaminated implant surface [25,26]. There is a consensus that correct oral hygiene should be established and that occlusal forces should be assessed and corrected by occlusal adjustment when deeply traumatic [26].

\section{Conclusion:-}

In the implant, if plate accumulation is allowed for longer periods and the patient is at risk, peri-implant mucositis can progress to lesions that extend more apically, with associated alveolar bone loss (peri-implantite). Due to the infectious nature of mucositis and peri-implantitis, preventive procedures should be performed in a control program and reassessed to ensure adequate supportive therapy for a lifetime. The most important treatment of peri-implantitis is to stop progressive bone loss by plaque control and purging, even restoring the bone lost through regenerative surgical procedures. 


\section{Competing Interests:-}

The authors declare que they have no competing interests.

\section{References:-}

1. Tavares LJ, Pavarina AC, Vergani CE, de Avila ED. The impact of antimicrobial photodynamic therapy on periimplant disease: What mechanisms are involved in this novel treatment? PhotodiagnosisPhotodynTher. 2016 Dec 6.pii: S1572-1000(16)30164-8. doi: 10.1016/j.pdpdt.2016.11.016.

2. King E, Patel R, Patel A, Addy L. Should implants be considered for patients with periodontal disease? Br Dent J. 2016 Dec 9;221(11):705-711. doi: 10.1038/sj.bdj.2016.905.

3. Kanwar IL, Sah AK, Suresh PK ${ }^{1}$.Biofilm-mediated Antibiotic-resistant Oral Bacterial. Curr Pharm Des. 2016 Nov 24.

4. Doornewaard R, Christiaens V, De Bruyn H, Jacobsson M, Cosyn J, Vervaeke S, Jacquet W. Long-Term Effect of Surface Roughness and Patients' Factors on Crestal Bone Loss at Dental Implants. A Systematic Review and MetaAnalysis. Clin Implant Dent Relat Res. 2016 Nov 15. doi: 10.1111/cid.12457.

5. John V, Shin D, Marlow A, Hamada Y. Peri-Implant Bone Loss and Peri-Implantitis: A Report of Three Cases and Review of the Literature. Case Rep Dent. 2016; 2491714. Epub 2016 Oct 19.

6. Del Amo F SL, Faria E Almeida R, Cicciù M, Daugela P, Ramanauskaite A, Saulacic N, Tervonen T, Wang HL, Yu SH. The 1st Baltic Osseointegration Academy and Lithuanian University of Health Sciences Consensus Conference 2016. Summary and Consensus Statements: Group III - Peri-Implantitis Treatment. J Oral Maxillofac Res. 2016 Sep 9;7(3):e16.

7. Lindhe, J. Et al. Experimental breakdown of pen-implant and periodontal tissues. A study in beagle dog. Clinical oral impints research. v.3 p. 9-16, 1992.

8. Mombelli, A. et al. Microbial aspects of implant dentistry. Periodontology 2000. v.4, p.74-80, 1994.

9. Gualine, F, Berglundh T. Immunohistochemicalcharasteristcs of inflammatory lesions at implants. J. Clin. Periodontol. v. 30 p. 14-18, 2003.

10. Esposito, M. et al. Immunohitochemistry of soft tissues surounding late failures of branemark implants. Clinical oral impints research. v. 8 P. 352-366, 1997.

11. Leljenberg, B. et al. Some microbiological, histopatological and immunohistochemicalcharacteristcs of progressive periodontal disease. J. Clin. Periodontol. v.21 p.720-727, 1994.

12. Branemark et al. "Close to the Edge - Brånemark and the Development of Osseointegration" - McClarence, Elaine Quintessence Books, cap. 1, Berlim, Alemanha, 2003).

13. Weyant, RJ, Burt, BA. An assessement of survival rates and within-patient clustering of failures for endosseus oral implants. J. dent. Res. v.72, p.2-8, 1993.

14. Pauleto, NC. et al. Effect of cigarette smoking on oral elastase ativity in adult periodontitis patients. J. periodontol. v.71, p.58-62, 2000.

15. Winkelhoff, V. et al. Smoking affects the subgigival microflora in periodontitis. J. periodontol. v.72, p. 666-671, 2001.

16. Bain CA, Moy PK. The association between the failure of dental implants and cigarette smoking. International J. of Oral and Maxilofacial Implants. v.8, p. 609-615, 1993.

17. Lindquist, LW et al. Association between marginal bone loss around osseintegrated mandibular implants and smoking habits. J. dent. Res. v.76, p.1667-1674, 1997.

18. Haas, R. et al. The relationship of smoking on pen-implant tissue.J. prosthetdent. v.76, p.592-596, 1996.

19. Fardal, O. et al. Severe, rapidly progressing peri-implantitis. J. din. Periodontol. v. 26, p. 313-317, 1999.

20. Quirynen, M. et al. Pen-implant health around screw-shaped c.p. titaniun machined implants in partially edentulous patients with or without ongoing periodontitis. Clin oral implants res. v.12 p.589-594, 2001.

21. Wowren, W. N., Symptomatic osteoporosis. J.prosther dent. v.67, p.656-660, 1992.

22. Wactawski et al. The role of osteopenia in oral bone loss and periodontal disease. J. periodontol. v.67, p.1076-1084, 1996.

23. Tailor GW. et al. Non-insulin dependent diabetes mellitus and alveolar bone loss progression over two years. J. periodontol. v. 69, p.76-83, 1998.

24. Ericsson, I. et al. Radiographical and histological charateristics of submerged and nonsubmerged titanium implants. An experimental study in the labrador dog. Clinical Oral Implants Research, Copenhagen, v.7, n.1, p.20-26, 1996.

25. Lang, N. P., et al. Biological complications with dental implants; their prevention, diagnosis and treatment. Clin oral impl. Res. 2000.,v.11, p. 146-155, 2000.

26. Behneke,A. Et al.Treatment of peri-implantitis defects with autogeous bone wafts International J. of Oral and Maxilofacial Implants v.15,p. 125-138. 2000. 